\title{
Antimicrobial Resistance Caused by KPC-2 Encoded by Promiscuous Plasmids of the Klebsiella pneumoniae ST307 Strain
}

\author{
Miyoung Lee $\mathbb{i}$, M.S. ${ }^{1,2}$ and Tae-Jin Choi $\mathbb{1}$, Ph.D. ${ }^{1}$ \\ ${ }^{1}$ Department of Microbiology, Pukyoung National University, Busan, Korea; ${ }^{2}$ Department of Laboratory Medicine, BHS Hanseo Hospital, Busan, Korea
}

Background: A lineage of Klebsiella pneumoniae that produces carbapenemase-2 (KPC2), sequence type (ST) 307, emerged in 2017. We analyzed the complete sequences of plasmids from KPC-2-producing K. pneumoniae (KPC-Kp) ST307, investigated the antimicrobial resistance conferred by this strain, and confirmed the horizontal interspecies transmission of KPC- carbapenemase-producing Enterobacteriaceae (CPE) characteristics among Enterobacteriaceae.

Methods: We performed antimicrobial susceptibility testing, PCR analysis, multilocus sequence typing, curing tests, and whole-genome sequencing to characterize plasmid-derived KPC-2-producing Enterobacteriaceae clinical isolates.

Results: Sequence analysis of KPC-Kp strain ST307 revealed novel plasmid-located virulence factors, including a gene cluster for glycogen synthesis. Three Enterobacteriaceae strains were identified in one patient: K. pneumoniae (CPKp1825), Klebsiella aerogenes (CPEa1826), and Escherichia coli (CPEc1827). The blakPc-2 gene from K. pneumoniae ST307 was horizontally transmitted between these strains. The plasmids could be transferred through conjugation, because all three strains of bacteria contained the type IV secretion system, pilus genes, and tra genes for conjugal transfer. The blakpc-2 gene was located on a truncated Tn4401 transposon. Plasmids containing the blakpc-2 gene could not be artificially removed; thus, the three strains could not be cured.

Conclusions: The ease of horizontal transfer of KPC-Kp ST307 carbapenem resistance has serious public health and epidemiological implications. This study provides a better understanding of the genetic characteristics that can contribute to the growth and spread of KPC-Kp ST307, and their association with antimicrobial resistance genes.

Key Words: Klebsiella pneumoniae, KPC-Kp ST307, blakpc gene, horizontal transfer, Tn4401, glycogen synthesis
Received: January 3, 2020

Revision received: April 10, 2020

Accepted: August 6, 2020

Corresponding author: Tae-Jin Choi, Ph.D. Department of Microbiology, Pukyoung National University, 45 Yongso-ro, Nam-gu, Busan 48513, Korea

Tel: +051-629-5617

Fax: +051-629-5619

E-mail: choitj@pknu.ac.kr

\section{INTRODUCTION}

The global spread of carbapenemase-producing (CP) Klebsiella pneumoniae (KPC) has become a major threat to human health [1]. Approximately 700,000 people die annually from antimicrobial-resistant infections, and this number is projected to surpass
10 million per year by 2050 [2]. This global phenomenon has been mainly associated with the dissemination of high-risk clones. The well-known KPC clones include the Kp sequence type (ST) 258 [3] and its related variants, which belong to clonal group 258 (CG258) [4]. However, in recent years, new drug-resistant lineages have emerged globally [5]. Among them, KPC-2-pro- 
ducing K. pneumoniae (KPC-Kp) ST307 is one of the most clinically relevant strains because its emergence has been recognized in several countries in the last five years [6, 7]. A study on non-CG258 clones of carbapenem-resistant $K$. pneumoniae (CR$\mathrm{Kp}$ ) strains spanning two yrs in Colombia showed that $62.2 \%$ of the strains were derived from ST307. Patients infected with KPCKp ST307 showed a high mortality rate (>50\%) and had longer hospital stays than patients infected with other clones, indicating that this lineage encodes additional factors that contribute to its virulence [8]. In addition, the ST307 genome encodes various pathogenic genetic characteristics. The highly mobile Tn3based transposon Tn4401 facilitates dissemination of the blaкрс gene [9]. The dissemination of blakpc is derived by the clonal spread of bacterial hosts harboring the gene or by horizontal transfer of transposons and plasmids carrying the gene [10,11]. IncX3-type plasmid is prevalent in K. pneumoniae carrying the blakpc gene [12].

As Kp ST307 is a relatively new strain, current knowledge on the genetic characteristics that facilitate its antimicrobial resistance and spread is limited. Thus, to enhance our understanding of Kp ST307 and facilitate the development of an effective therapy against infections caused by this strain, we investigated the genetic characteristics of KPC-Kp ST307 that contribute to its growth and spread, its antimicrobial resistance, and transfer among different Enterobacteriaceae strains. Additionally, we performed a whole-genome sequence analysis of KPC-Kp ST307 and other bacterial strains isolated from a single patient and investigated the horizontal transfer of plasmids containing the promiscuous blakpc gene across intestinal bacterial strains of the patient. The whole-genome sequences were then compared and analyzed using minimum inhibitory concentration (MIC) analysis.

\section{MATERIALS AND METHODS}

\section{Sources of KPC-producing Enterobacteriaceae isolates}

The Institutional Review Board of BHS Hanseo Hospital, Busan, Korea, approved this prospective study (approval number: CTS19-003). An 87-year-old woman with a history of primary knee arthropathy in both knees was admitted to a general hospital in Busan in August 2018 for arthritis treatment. CR Enterobacteriaceae were not detected in a rectal swab at admission. However, after one month, a urine culture test performed because of fever, confirmed KPC-2-producing Kp (CPKp1825). Additionally, Klebsiella aerogenes (CPEa1826) and Escherichia coli (CPEc1827) were detected from rectal swabs at 23 and 26 days, respectively, following the detection of the CPKp1825 strain in the urine cul- ture. After the isolation of CPKp1825, vancomycin-resistant Enterococcus faecium was isolated from urine cultures repeated once every three days for two months, and the stool tested positive for Clostridioides difficile toxin.

\section{Bacterial isolates and antimicrobial susceptibility testing}

We identified clinical isolates using standard microbiological procedures and the identity of all isolates was confirmed by $16 \mathrm{~S}$ rRNA gene sequencing [13]. Antimicrobial susceptibilities were determined by VITEK 2 AST N224 cards (bioMérieux Vitek, Hazelwood, MO, USA); susceptibility against 12 antimicrobial agents —ampicillin, aztreonam, cefotaxime, ceftazidime, cefoxitin, ciprofloxacin, amikacin, gentamicin, imipenem, meropenem, ertapenem, and colistin — was evaluated by the disk diffusion method on Mueller-Hinton (MH) agar (Becton Dickinson, Franklin Lakes, NJ, USA), following the Clinical and Laboratory Standards Institute (CLSI) guidelines [14]. Colistin susceptibility was determined by the broth microdilution method with freshly prepared cationadjusted MH broth, following the CLSI guidelines [14]. Tigecycline susceptibility was confirmed by an E-test (bioMérieux, Marcy-l'Etoile, France). For the modified carbapenem inactivation method ( $\mathrm{mClM}$ ) test, the bacteria were incubated with a meropenem disk in tryptic soy infusion (TSI; Difco Laboratories, Detroit, MI, USA) broth. For the EDTA-modified carbapenem inactivation method (eCIM) test, EDTA was added to the broth to chelate metal ions necessary for the metallo- $\beta$-lactamase function. After incubation, the disks were removed and placed on a lawn of susceptible $E$. coli to determine whether the test bacteria hydrolyze meropenem. Zone diameters were measured and interpreted following the CLSI guidelines [14].

\section{Detection of resistance genes}

We screened Kp strains for genes encoding extended-spectrum $\beta$-lactamases (ESBLs) (blactx-м-1, blactх-м-9., blaтем-, and blasнvtype), carbapenemases (bla IMP-1-type, blaviм-2-type, blandm, blakPC, blakPC-2, blaGEs, and blaoxA-48-ike) [15], and outer membrane proteins (ompK35 and ompK36) using PCR (Verity 96-Well; Applied Biosystems, Eatate, Singapore) [16]. The PCR products were sequenced using a 3730xl automatic sequencer (Applied Biosystems, Weiterstadt, Germany), and the nucleotide sequences were compared using the Basic Local Alignment Search Tool (https://blast.ncbi.nlm.nih.gov/Blast.cgi) (Supplemental Data Table S1).

\section{Multilocus sequence typing (MLST)}

We performed MLST PCR and sequencing for seven housekeep- 
ing genes (gapA, infB, $m d h$, pgi, phoE, rpoB, and ton $B$ for $\mathrm{Kp}$ [17], and adk, fumC, gyrB, icd, $m d h$, purA, and recA for E. coli) [18]. Nucleotide sequences obtained for both DNA strands were compared with sequences in the MLST database for each species (http://bigsdb.web.pasteur.fr/klebsiella for $\mathrm{Kp}$ and http:// mlst.warwick.ac.uk/mlst/dbs/Ecoli for E. coli), to determine allelic numbers and STs.

\section{Bacterial conjugation}

Plasmids carrying the blakpc gene in the CPKp1825, CPEa1826, and CPEc1827 strains were used as donors, and sodium azideresistant $E$. coli J53 was used as the recipient [19]. For the standard agar mating method, equal amounts of overnight donor and recipient cultures were mixed and immediately spread on brain heart infusion (BHI; MBcell, Los Angeles, CA, USA) agar. After overnight mating at $37^{\circ} \mathrm{C}$, bacterial cells were recovered and plated on MacConkey agar containing sodium azide (100 $\mu \mathrm{g} / \mathrm{mL})$ and imipenem $(0.5 \mu \mathrm{g} / \mathrm{mL})$ to select transconjugants.

\section{Curing test}

We performed KPC gene-carrying plasmid curing using the temperature-mediated plasmid emission method. CR Enterobacteriaceae strains were subjected to elevated temperature-mediated plasmid elimination through sequential passages in $\mathrm{BHI}$ (MBcell) broth twice per day for two weeks. Thereafter, the cultures were diluted and plated on TSI medium to obtain single colonies. Suspected cured colonies were identified by picking and plating onto TSI medium containing $5 \mu \mathrm{g} / \mathrm{mL}$ of imipenem and onto control TSI medium lacking imipenem. Colonies that failed to grow in the presence of imipenem were suspected to be cured and were further analyzed for blakpc loss by PCR and antimicrobial susceptibility testing [20].

\section{Whole-genome sequencing (WGS)}

We performed WGS of the CPKp1825, CPEa1826, and CPEC1827 isolates using single-molecule real-time sequencing on a PacBio RSII instrument (Pacific Biosciences, Menlo Park, CA, USA). Schematic diagrams of multiple plasmid alignments were prepared by manually realigning the linear plasmid maps drawn using SnapGene Viewer (http://www.snapgene.com/products/ snapgene viewer/). Genomes were compared using Mauve comparison tools (http://darlinglab.org/mauve/). Resistance genes, insertion sequence elements, replication origins, virulent elements, and toxin and antitoxin systems were identified using the following databases: ResFinder (https://cge.cbs.dtu.dk//services/ ResFinder/), ISfinder (https://www-is.biotoul.fr/), plasmid finder

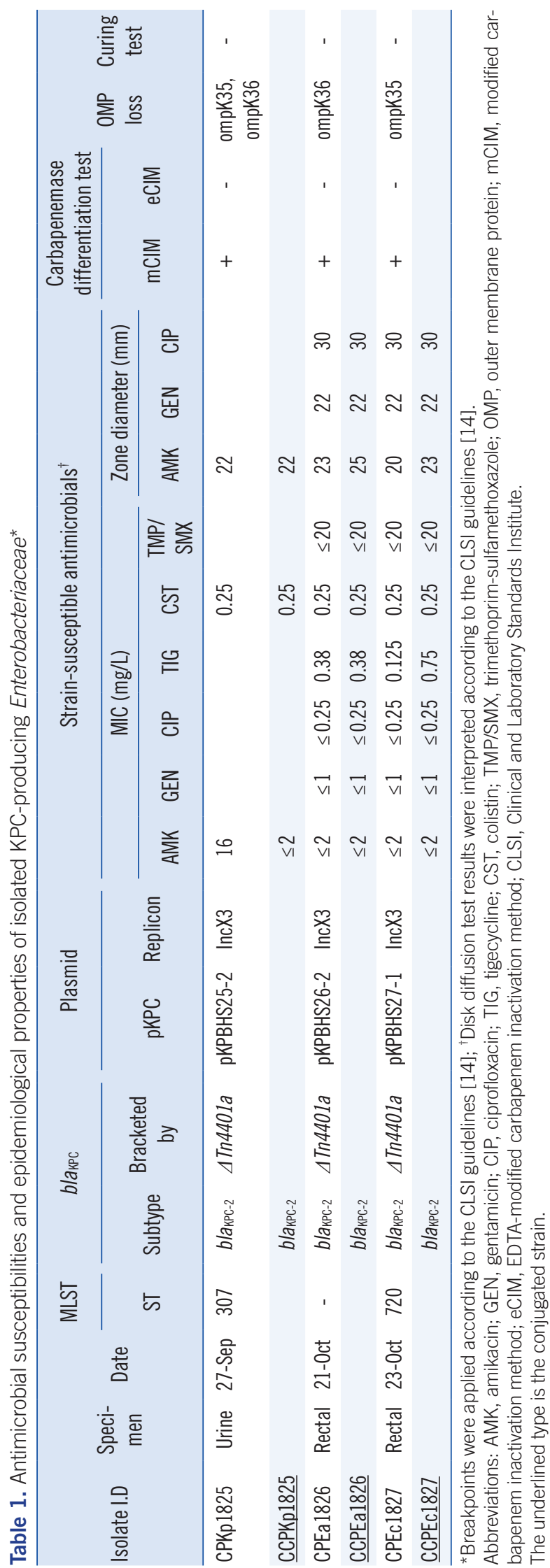


(https://cge.cbs.dtu.dk/services/PlasmidFinder/), RestrictionModification finder (https://cge.cbs.dtu.dk/services/RestrictionModificationFinder//), Serotype finder (https://cge.cbs.dtu.dk/ services/SerotypeFinder/), Fim Typer (https://cge.cbs.dtu.dk/ services/FimTyper/), and CH Typer (https://cge.cbs.dtu.dk/services/CHTyper/).

\section{GenBank accession numbers}

Nucleotide sequence data for CPKp1825, CPEa1826, and CPEc1827 are available under GenBank accession numbers WMHT01000001-WMHT01000003, WMHU01000001-WMHU01000003, and WMHV01000001-WMHV01000003, respectively.

\section{RESULTS}

Antimicrobial susceptibilities and molecular typing The Kp (CPKp1825) isolates showed a multidrug resistance (MDR) phenotype. The isolates exhibited resistance to most antimicrobials tested, including ampicillin, aztreonam, cefotaxime, ceftazidime, cefoxitin, ciprofloxacin, gentamicin, tigecycline, imipenem, meropenem, and ertapenem, but were susceptible to amikacin and colistin. K. aerogenes (CPEa1826) and E. coli (CPEc1827) isolates were resistant to ampicillin, aztreonam, cefotaxime, ceftazidime, cefoxitin, imipenem, meropenem, and ertapenem, but were susceptible to amikacin, gentamicin, tigecycline, ciprofloxacin, trimethoprim/sulfamethoxazole, and colistin. The susceptibility test results for combinations of $\beta$-lactamases and sites of isolation for the isolates assessed are shown in Table 1.

PCR and sequencing of $\beta$-lactamase genes revealed the presence of blakpc-2 in all isolates. SHV-1-like broad-spectrum penicillinase, which is intrinsic, was identified by PCR in both CPKp1825 and CPEa1826 isolates. Genes for plasmid-mediated CTX-M-type ESBLs were found in CPKp1825 isolates. In addition, we found that OmpK35 and OmpK36 were missing from CPKp1825 isolates, OmpK36 was missing from CPEa1826 isolates, and OmpK35 was missing from CPEc1827 isolates. Plasmids carrying the blakPC-2 gene in CPKp1825, CPEa1826, and CPEc1827 could be transferred to E. coli J53. The conjugated strains had the same resistance gene as that of the pre-junction strain (Table 2). A curing test was performed to artificially remove plasmids containing the KPC-2 gene. However, none of the three strains could be successfully cured (Table 1 ).

\section{Sequencing and annotation of CPKp1825}

The Kp strain CPKp1825 has a 5,812,656-bp genome, comprising of a single chromosome and two plasmids (pKPBHS_25_1 and pKPBHS_25_2). The CPKp1825 chromosome consisted of

Table 2. Resistome and integrative conjugative elements

\begin{tabular}{|c|c|c|c|c|c|c|c|c|c|}
\hline \multirow[b]{2}{*}{ Strain } & \multirow{2}{*}{$\begin{array}{l}\text { Plasmid } \\
\text { Replicon }\end{array}$} & \multicolumn{7}{|c|}{ Antimicrobial resistance gene } & \multirow[b]{2}{*}{ Conjugants } \\
\hline & & $\beta$-lactam & $\begin{array}{l}\text { Aminogly- } \\
\text { coside }\end{array}$ & Tetracycline & Macrolide & Phenicol & $\begin{array}{l}\text { Trime- } \\
\text { thoprim }\end{array}$ & Sulfonamide $\begin{array}{c}\text { Fluoroquino- } \\
\text { lone }\end{array}$ & \\
\hline ССРКр1825 & & $\begin{array}{c}\text { blaкрс-2, blaстх-м-1, } \\
\text { blasнv, blaтем-1 }\end{array}$ & $r m t B$ & & & & & & \\
\hline & $\operatorname{lncX3}$ & $\begin{array}{l}\text { blaкPC-2, blaстх-м-15, } \\
\text { blasнV-182, blaтем-1в }\end{array}$ & $\begin{array}{c}\text { aac(3)-lia, } \\
\text { aph(3")-Ib, } \\
\text { aph(6)-ld }\end{array}$ & & & & dfrA14 & sul2 & \\
\hline CPEa1826 & & blakPC-2, blasнv, & & & & & & & + \\
\hline CPEc1827 & & blaкPс-2 & & & & & & & + \\
\hline CCPEс $1827^{\dagger}$ & & blaкрс-2 & & & & & & & \\
\hline pECBHS_27_1 & $\operatorname{lnc} X 3$ & blaкрс-2 & & & & & & & \\
\hline
\end{tabular}

Whole-genome sequencing was performed for the indicated plasmids. CPKp1825, CPEa1826, and CPEc1827 are the pre-conjugation strains, and CCP$\mathrm{Kp} 1825^{\dagger}$, CCPEa $1826^{\dagger}$, and CCPEc $1827^{\dagger}$ are the conjugated strains, which were analyzed using DNA sequencing. 
a 5,541,320-bp circular DNA molecule with an average G+C content of 57.2\% and harbored 5,143 annotated open reading frames (ORFs). We found no known mutations, but unknown mutations in the genomes included amino acid substitutions P161R, G164A, F172S, R173G, L195V, F197I, and K201M in AcrR. L188Q and K194* substitutions in RamR, OmpK36, and OmpK37 were also found. The type II restriction enzyme M.Kpn$34618 D \mathrm{~cm}$ was also present. CPKp1825 was identified as an ST307 strain. The chromosome of CPKp1825 isolates included blashv-28 and blashv-106 for $\beta$-lactam resistance, oqXA, B for quinolone resistance, and fos $A$ for fosfomycin resistance.

The multi-replicon (IncFIB (K) and IncFII (K)) plasmid pKPBHS_25_1 included aac (6')-Ib-cr for fluoroquinolone and aminoglycoside resistance, blaoxA-1 for $\beta$-lactam resistance, and catB3 for phenicol resistance (Fig. 1A). Further, pKPBHS_25_1 encoded CusCFBA proteins that mediate resistance to copper and silver by cation efflux, and carried five putative virulence clusters, the lacZYI operon, the Fec-like iron (III) dicitrate and glutathione ABC-transport systems, the urea transport system, and a cluster for glycogen synthesis. A 35-kb region on pKPBHS_25_1 contains a type IV secretion system, the pilus genes, and tra genes for conjugal transfer (Fig. 1B).

pKPBHS_25_2 (72,849 bp), an incompatibility group $X$ type 3 (IncX3) plasmid, was found to belong to an incompatibility group (Fig. 1C) and carry genes encoding type II restriction enzymes Eco128I and M.EcoRII. The plasmid pKPBHS_25_2 contained aac (3)-Ila, aph (3')-Ib, and aph (6)-Id for aminoglycoside resistance; blactx-M-15, blakpc-2, blasнV-182, and blaтем-1в for $\beta$ lactam resistance; sul2 for sulfonamide resistance; and dfrA14 for trimethoprim resistance. The blakpc-2 gene was located on a truncated Tn4401 transposon. $\Delta$ ISKpn7-blakpc-2-ISKpn6 had a 99-bp deletion between ISKpn7 and blakpc, indicating that it is a Tn4401a isoform. The plasmid was found to carry the Tn3 transposon containing blaтем-1в. A 13-kb region on pKPBHS25-2 contained a type IV secretion system, the pilus genes, and tra genes for conjugal transfer (Fig. 1C and Fig. 2).

\section{Sequencing and annotation of CPEa1826}

WGS revealed that the $K$. aerogenes strain CPEa1826 has a 5,254,026-bp genome with two plasmids (pEABHS_26_1 and pEABHS_26_2). The nucleotide sequence variations of unknown mutations in 23S, 16S rsH, B, C, and amino acid change of rpoB were confirmed. M.SenAnal, the methyltransferase type I restriction enzyme, was also detected. The chromosome CPEa1826 did not contain any acquired antimicrobial resistance determinants. Plasmid pEABHS_26_1, belonging to the IncP-1 group,
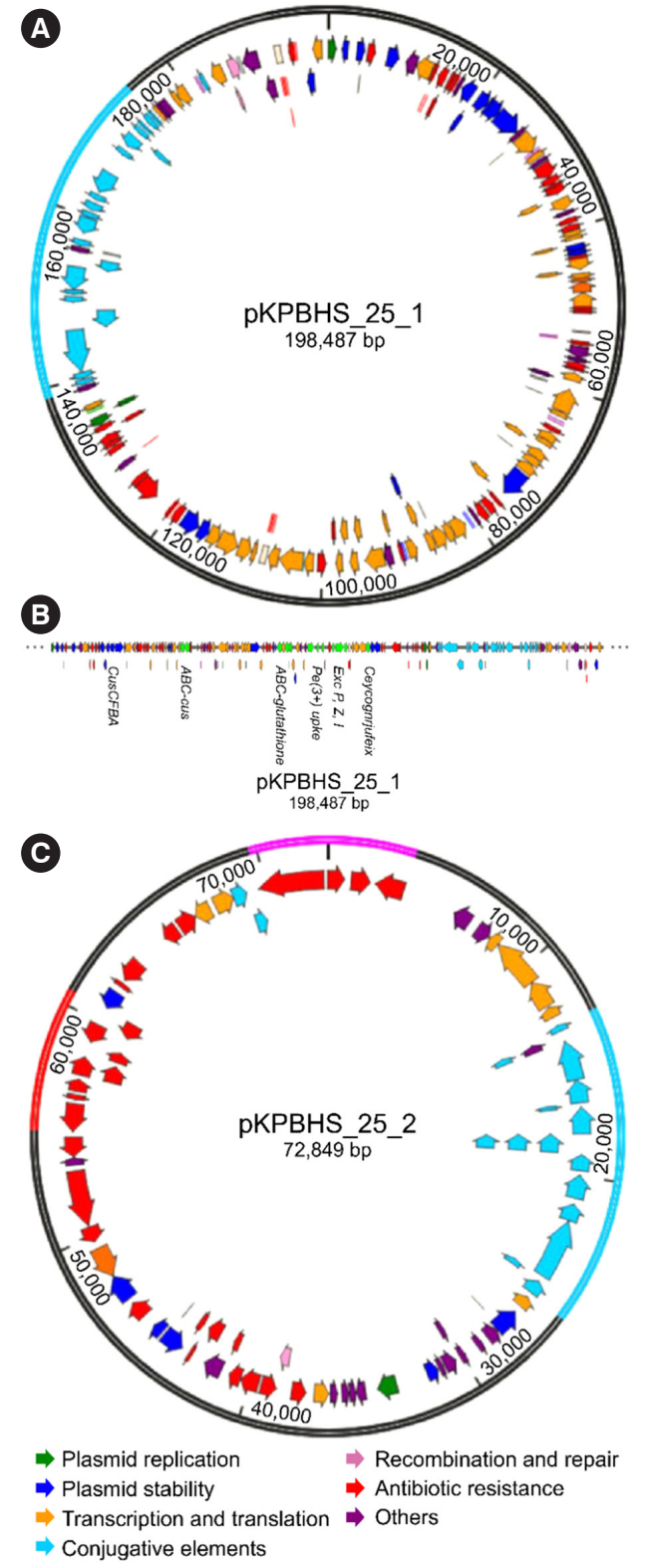

Fig. 1. Genetic organization of plasmids associated with blakpc. (A) Circular map of pKPBHS_25_1 with two replication origins for the IncFIB (K) and IncFIl (K) groups, as well as a copper-transporting efflux system (CusCFBA) and five putative virulence clusters. (B) Variant pKPBHS_25_1 plasmids identified in ST307. (C) Circular map of pECBHS_25_2 containing $\triangle T$ Tn4401a with blactx-M-15, blakPC-2, blasнV-182, blaтем-1в, and conjugative elements. Each arrow indicates plasmid scaffold genes and their direction of transcription. The locus Tra is indicated by sky blue arrows, indicating the tra genes (e.g. traG, G; traF, F; traO, O). Related genes (tnpA, tnpR, and tnpM), resistance genes, and insertion sequences are indicated by red arrows. Other genes are indicated by colored arrows as follows: orange, transcription, and translation genes; blue, CusCFBA; green, clusters encoding putative virulence determinants. In the plasmid circle, the Tn3 transposon is indicated in red, $\Delta \mathrm{Tn} 4401 \mathrm{a}$ with blakpc-2 in pink and conjugative elements in sky blue. 


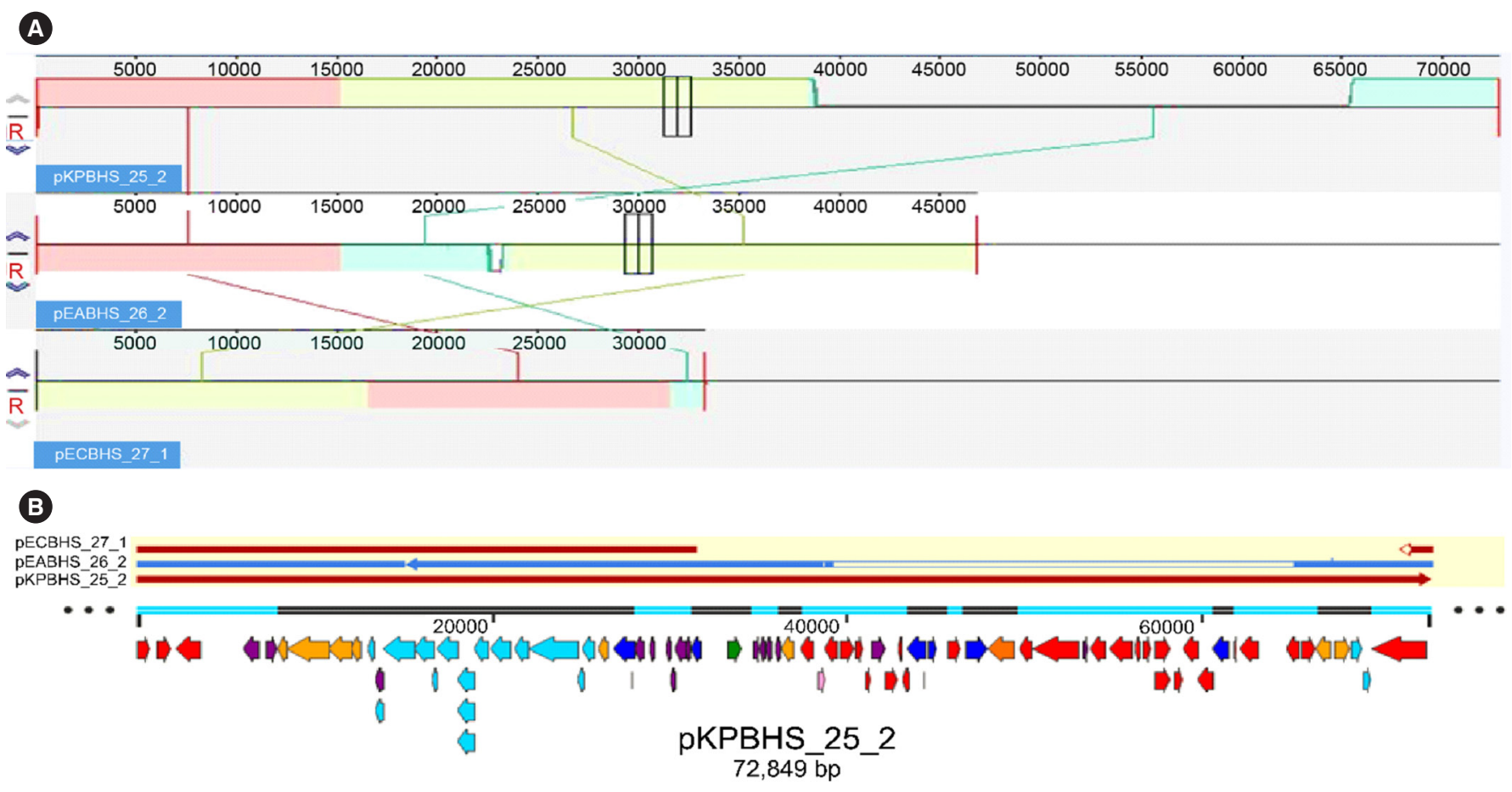

Fig. 2. Linear comparison of the genetic surroundings of blaкрс-2. (A) One fragment unit shows pink, yellow green, and green on the contig. Gene contents were similar along the synteny on the contig. The fragment was well preserved, but inversion existed. Most genes were well preserved. (B) pKPBHS_25_2, pEABHS_26_2, and pECBHS_27_1 are denoted by arrows and colored based on gene-functional classification. Other genes are indicated by colored arrows as follows: green, plasmid replication; blue, plasmid stability; orange, transcription and translation; sky blue, conjugative elements; pink, recombination and repair; red, antimicrobial resistance; purple, other genes.

did not contain any acquired antimicrobial resistance gene, and encoded duplicated type IV secretion systems and conjugative elements. The 46,836-bp plasmid pEABHS_26_2 carrying the blakPc-2 and blasHv-182 genes belongs to the IncX3 group. pEABHS_ $26 \_2$ had an average $\mathrm{G}+\mathrm{C}$ content of $48.0 \%$ and harbored 65 annotated ORFs. The blakpc-2 gene was located on a truncated Tn4401 transposon (Fig. 2 and Fig. 3A).

\section{Sequencing and annotation of CPEc1827}

The E. coli strain CPEc1827 was found to have a 5,033,400-bp genome comprising a single chromosome and a plasmid. The genome had a S57T substitution in ParC. The nucleotide sequence variations of unknown mutations of 16S_rrsB, $\mathrm{H}, \mathrm{C}$, and 23S, and amino acid changes in pmrB and parC, as well as the nucleotide sequence variations of unknown mutations of the 23S, $16 \mathrm{~S} \mathrm{rsH}, \mathrm{B}, \mathrm{C}$, and amino acid changes in rpoB were confirmed. Type II restriction enzymes M.EcoE455Dcm and M.EcoGVI were also found. CPEC1827 was identified as ST720 and belonged to the serotypes ST17/O77 and H41, while its subtype to fum C3 and fim H65. The chromosome included $m d f(A)$ for macrolide, lincosamide, and streptogramin B resistance. The 91,356-bp contig did not contain any acquired antimicrobial resistance determinants. The 33,268-bp IncX3 plasmid (pECBHS_27_1) carried blakрс-2 for $\beta$-lactam resistance (Fig. 3B). The blaкрс-2 gene was found to be located on a truncated Tn4401 transposon, and conjugal transfer genes (including tra and trb) were located at 12,738 bp (Fig. 2 and 3B).

\section{DISCUSSION}

KPC-Kp ST307 is a novel lineage that has the potential to become an epidemic or 'high-risk' clone. ST258 is a well-known KPC-producing clone with a global distribution [3, 4]. However, according to the literature, it is not prevalent in Korea [21]. The most common clone in Korea is KPC-Kp ST307. Several reports have indicated local dissemination of ST307 harboring the blakPc genes blakpc-2 (Columbia, USA, Korea) and blakpc-s (Italy) [8, 22, 23], whereas an analysis of 1,700 ESBL-producing Kp from a hospital network in Texas, USA revealed a high prevalence of blactx-M-15-positive ST307 strains, with one-third of the strains also carrying blaкрс-2 genes and only three carrying blaкрс-3 [24].

The blactx-M-15 gene is common in ST307 strains [21, 24, 25]. 

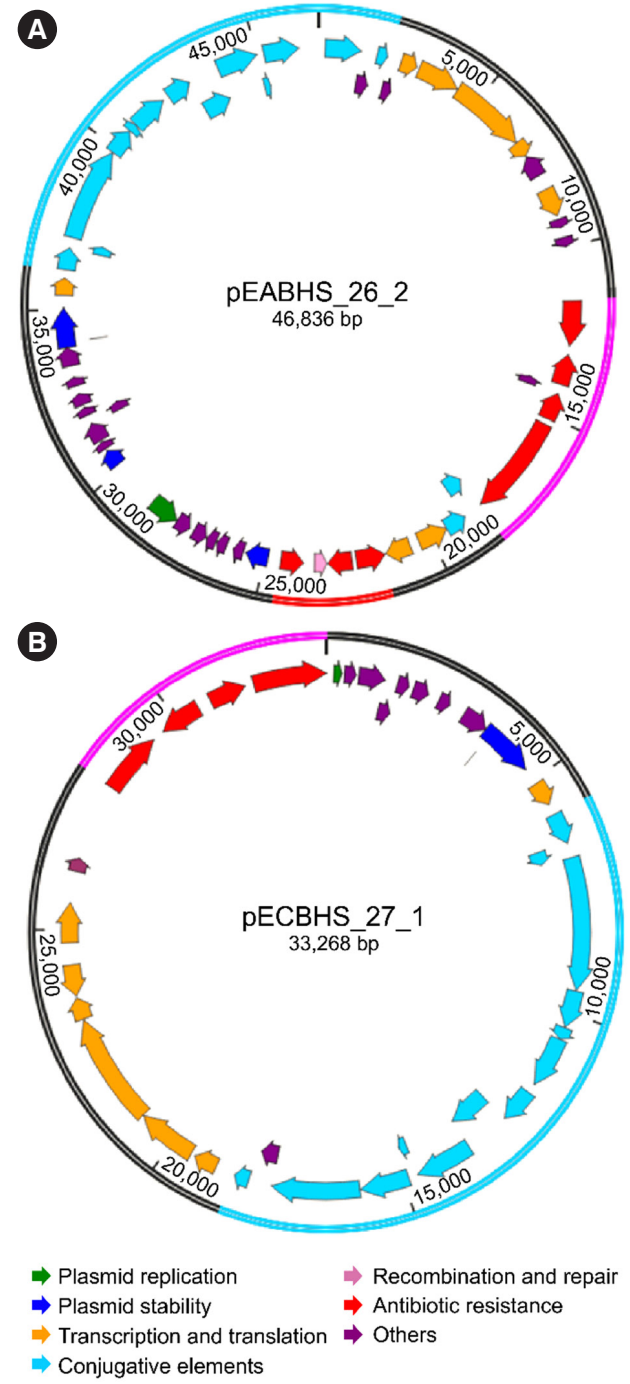

Fig. 3. Genetic organization of plasmids pEABHS_26_2 and pECBHS_27_1. (A) Circular map of pEABHS_26_2 containing $\triangle T n 4401 a$ harboring blakPC-2 and blasHV-182 genes. (B) Circular map of pECBHS_27_1 containing $\triangle \mathrm{Tn} 4401 \mathrm{a}$ harboring blakPC-2 and conjugal transfer genes. In the plasmid circle, the Tn3 transposon is indicated in red, $\triangle T$ T4401a with blaкрс-2 in pink and conjugative elements in sky blue.

According to Wyres, et al. [26], ParC 80I and GyrA 83I fluoroquinolone resistance-associated mutations were conserved in all 95 genomes analyzed in their study. The study also showed the blactx-M-15 ESBL gene to be present in 89 genomes (93.7\%), and 81 genomes (85.3\%) harbored sul2, dfrA14, and strAB with or without aac(3)-Ila, which were all linked to an MDR plasmid. These results are consistent with our findings. In addition to blactх-м-15, blasнV-182 and blaтем-1в were repeatedly detected during the 38-day hospitalization period of the patient, resulting in continuous infection. Thus, plasmid-mediated glycogen syn- thesis might help ST307 isolates survive under limited nutrient availability, and the urea transport system might facilitate colonization of this strain in the urinary tract.

Type II restriction enzymes were observed in CR-KP1725 and CR-KP1727. Type II restriction enzymes function to protect their host DNA by removing extracellular foreign DNA that enters the cell to maintain its pathological state. The presence of different promoters, leading to various levels of expression of the type II restriction enzymes, could be responsible for the variability in resistance levels between CR-KP1725 and CR-KP1727, but this might not be sufficient to explain the high level of resistance, as previously suggested [27].

All three strains had lost their outer membrane proteins. The strain lacking OmpK36 alone became resistant to cefazolin, cephalothin, and cefoxitin, indicating that the disruption of ompK36 results in greater drug resistance than the disruption of ompK35. The additional loss of OmpK35 further increased the MICs, which demonstrated that the double-deletion strain was highly resistant to the antimicrobials [28]. All these genetic functions synergistically make KP ST307 more resistant to antimicrobials and more difficult to treat in patients. The MIC results for amikacin in this study should be interpreted cautiously [29]. Sensitivity was confirmed in MIC and antimicrobial disk tests, but WGS analysis revealed that genes including aac (3)-Ila, aph (6)-Id, and aph (3")-Ib were resistance genes. Only 3\% (2/65) and $11 \%(7 / 65)$ of the isolates were non-susceptible to amikacin using the CLSI and EUCAST breakpoints, respectively [14, 30]. These low rates were observed even though 73\% (16/22) of aminoglycoside-modifying enzyme (AME)+isolates possessed aac (6)-Ib, which is reported to confer amikacin resistance. Amikacin MICs below the susceptibility breakpoints are commonly observed among Enterobacter and other Enterobacteriaceae that possess aac $\left(6^{\prime}\right)-I b[29,31]$. The clinical significance of low MICs against bacteria carrying AMEs that are known to cause resistance has not been established, and it is unclear whether MICs or molecular markers are more relevant to treatment responses [32]. Indeed, the EUCAST states that should a member of the Enterobacteriaceae test as tobramycin (TOB)-intermediate or TOB-resistant and gentamicin- and amikacin-susceptible, its amikacin susceptibility status should be revised to "intermediate" [30]. Until the clinical significance of these low MICs is resolved, we recommend that amikacin be used with caution in the treatment of infections caused by Enterobacter that are resistant to other aminoglycosides.

Our study revealed that the blakPC-2 gene from KPC-Kp ST307 could be horizontally transferred to other bacterial species. The 
blakPC-2 gene of KPC-Kp ST307 is encoded by a conjugable plasmid, which can be speculated to provide a possible mechanism for dissemination to other species of gram-negative bacilli. Plasmids from all three strains could be transmitted through conjugation. Tn4401, a transposable element, could also move across the species. This shows the limitation of symptomatic treatment, as well as the risk posed by KPC-Kp ST307. CR Enterobacteriaceaeinfected patients have a high mortality rate, and the therapeutic agents used to treat their condition are limited. Thus, treatment of CR Enterobacteriaceae infection often involves a combination therapy, including carbapenem, aminoglycosides, and fosfomycin. Monotherapies of colistin or tigecycline are the last line of treatment; however, this therapeutic option is not yet available in Korea.

This study had two limitations. First, only KPC-producing Enterobacteriaceae strains observed at admission were analyzed, and molecular microbiological evidence was not generated for carbapenemase resistance in Enterobacteriaceae infections that occurred later, which limits the generalizability of our findings. Second, an epidemiological study of the KPC-Kp isolates to identify their origin of occurrence was not conducted. However, the ease of horizontal transfer of KPC-Kp ST307 plasmids observed in this study has serious public health and epidemiological implications. MDR Kp ST307 should be monitored alongside other well-known clones to detect emerging MDR threats. Our findings indicate an urgent need for enhanced surveillance of KPCKp ST307. In particular, the occurrence of KPC-Kp ST307 in Korea, with its high frequency, suggests a health threat. Controlling the spread and limiting the impact of KPC-Kp ST307 isolates in Korean hospitals will require intensive efforts in both public and private healthcare centers. These data may serve an important role in understanding and controlling the spread of CR gram-negative pathogens.

\section{ACKNOWLEDGMENTS}

We thank BHS Hanseo Hospital for kindly accommodating us and allowing us to continue our study.

\section{AUTHOR CONTRIBUTIONS}

MYL performed experiments. MYL and TJC analyzed the data and wrote the manuscript.

\section{CONFLICTS OF INTEREST}

None declared.

\section{RESEARCH FUNDING}

None declared.

\section{ORCID}

Miyoung Lee

https://orcid.org/0000-0003-0322-0415

Tae-Jin Choi https://orcid.org/0000-0002-4923-5121

\section{REFERENCES}

1. Yoon EJ, Oh Y, Jeong SH. Development of tigecycline resistance in carbapenemase-producing Klebsiella pneumoniae sequence type 147 via AcrAB overproduction mediated by replacement of the ramA promoter. Ann Lab Med 2020;40:15-20.

2. O'Neill J. Antimicrobial resistance: tackling a crisis for the health and wealth of nations. London: Review of antimicrobial resistance. https:// amr-review.org/sites/default/files/AMR\%20Review\%20Paper\%20-\%20 Tackling\%20a\%20crisis\%20for\%20the\%20health\%20and\%20wealth \%20of\%20nations_1.pdf (Updated on Dec 2016).

3. Pitout JD, Nordmann P, Poirel L. Carbapenemase-producing Klebsiella pneumoniae, a key pathogen set for global nosocomial dominance. Antimicrob Agents Chemother 2015;59:5873-84.

4. Deleo FR, Chen L, Porcella SF, Martens CA, Kobayashi SD, Porter AR, et al. Molecular dissection of the evolution of carbapenem-resistant multilocus sequence type 258 Klebsiella pneumoniae. Proc Natl Acad Sci U S A 2014;111:4988-93.

5. Wyres KL and Holt KE. Klebsiella pneumoniae population genomics and antimicrobial-resistant clones. Trends Microbiol 2016;24:944-56.

6. Girlich D, Bouihat N, Poirel L, Benouda A, Nordmann P. High rate of faecal carriage of extended-spectrum $\beta$-lactamase and OXA-48 carbapenemase-producing Enterobacteriaceae at a university hospital in Morocco. Clin Microbiol Infect 2014;20:350-4.

7. Castanheira M, Farrell SE, Wanger A, Rolston KV, Jones RN, Mendes RE. Rapid expansion of KPC-2-producing Klebsiella pneumoniae isolates in two Texas hospitals due to clonal spread of ST258 and ST307 lineages. Microb Drug Resist 2013;19:295-7.

8. Ocampo AM, Chen L, Cienfuegos AV, Roncancio G, Chavda KD, Kreiswirth BN, et al. A two-year surveillance in five Colombian tertiary care hospitals reveals high frequency of non-CG258 clones of carbapenemresistant Klebsiella pneumoniae with distinct clinical characteristics. Antimicrob Agents Chemother 2015;60:332-42.

9. He S, Chandler M, Varani AM, Hickman AB, Dekker JP, Dyda F. Mechanisms of evolution in high-consequence drug resistance plasmids. MBio 2016; 7:e01987-16.

10. Lee CR, Lee JH, Park KS, Kim YB, Jeong BC, Lee SH. Global dissemination of carbapenemase-producing Klebsiella pneumoniae: epidemiology, genetic context, treatment options, and detection methods. Front Microbiol 2016;7:895.

11. Chen YT, Lin JC, Fung CP, Lu PL, Chuang YC, Wu TL, et al. KPC-2-encoding plasmids from Escherichia coli and Klebsiella pneumoniae in Taiwan. J Antimicrob Chemother 2014;69:628-31.

12. Johnson TJ, Bielak EM, Fortini D, Hansen LH, Hasman H, Debroy C, et al. Expansion of the IncX plasmid family for improved identification and typing of novel plasmids in drug-resistant Enterobacteriaceae. Plasmid 2012;68:43-50.

13. Cicek AC, Duzgun AO, Saral A, Sandalli C. Determination of a novel in- 
tegron-located variant (blaOXA-320) of class D $\beta$-lactamase in Proteus mirabilis. J Basic Microbiol 2014;54:1030-5.

14. CLSI. Methods for dilution antimicrobial susceptibility tests for bacteria that grow aerobically. 11th ed. CLSI M07. Wayne, PA: Clinical and Laboratory Standards Institute, 2018.

15. Jeong S, Kim JO, Jeong SH, Bae IK, Song W. Evaluation of peptide nucleic acid-mediated multiplex real-time PCR kits for rapid detection of carbapenemase genes in gram-negative clinical isolates. J Microbiol Methods 2015;113:4-9.

16. Landman D, Bratu S, Quale J. Contribution of ompK36 to carbapenem susceptibility in KPC-producing Klebsiella pneumoniae. J Med Microbiol 2009;58:1303-8.

17. Diancourt L, Passet V, Verhoef J, Grimont PA, Brisse S. Multilocus sequence typing of Klebsiella pneumoniae nosocomial isolates. J Clin Microbiol 2005;43:4178-82.

18. Wirth T, Falush D, Lan R, Colles F, Mensa P, Wieler LH, et al. Sex and virulence in Escherichia coli: an evolutionary perspective. Mol Microbiol 2006;60:1136-51.

19. Jeong SH, Lee KM, Lee J, Bae IK, Kim JS, Kim HS, et al. Clonal and horizontal spread of the blaOXA-232 gene among Enterobacteriaceae in a Korean hospital. Diagn Microbiol Infect Dis 2015;82:70-2.

20. Leavitt A, Chmelnitsky I, Ofek I, Carmeli Y, Navon-Venezia S. Plasmid pKpQIL encoding KPC-3 and TEM-1 confers carbapenem resistance in an extremely drug-resistant epidemic Klebsiella pneumoniae strain. J Antimicrob Chemother 2010;65:243-8.

21. Yoon EJ, Kim JO, Kim D, Lee H, Yang JW, Lee KJ, et al. Klebsiella pneumoniae carbapenemase producers in South Korea between 2013 and 2015. Front Microbiol 2018;9:56.

22. Bonura C, Giuffrè M, Aleo A, Fasciana T, Di Bernardo F, Stampone T, et al. An update of the evolving epidemic of blaKPC carrying Klebsiella pneumoniae in Sicily, Italy, 2014: emergence of multiple non-ST258 clones. PLoS One 2015; 10:e0132936.

23. Yoon EJ, Yang JW, Kim JO, Lee H, Lee KJ, Jeong SH. Carbapenemaseproducing Enterobacteriaceae in South Korea: A report from the National Laboratory Surveillance System. Future Microbiol 2018;13:771-
83.

24. Long SW, Olsen RJ, Eagar TN, Beres SB, Zhao P, Davis JJ, et al. Population genomic analysis of 1,777 extended-spectrum beta-lactamaseproducing Klebsiella pneumoniae isolates, Houston, Texas: Unexpected abundance of clonal group 307. mBio 2017;8:e00489-17.

25. Villa L, Feudi C, Fortini D, Brisse S, Passet V, Bonura C, et al. Diversity, virulence, and antimicrobial resistance of the KPC-producing Klebsiella pneumoniae ST307 clone. Microb Genom 2017;3:e000110.

26. Wyres KL, Hawkey J, Hetland MAK, Fostervold A, Wick RR, Judd LM, et al. Emergence and rapid global dissemination of CTX-M-15-associated Klebsiella pneumoniae strain ST307. J Antimicrob Chemother 2019; 74:577-81.

27. Roth AL, Kurpiel PM, Lister PD, Hanson ND. blakPC RNA expression correlates with two transcriptional start sites but not always with gene copy number in four genera of gram-negative pathogens. Antimicrob Agents Chemother 2011;55:3936-8.

28. Tsai YK, Fung CP, Lin JC, Chen JH, Chang FY, Chen TL, et al. Klebsiella pneumoniae outer membrane porins OmpK35 and OmpK36 play roles in both antimicrobial resistance and virulence. Antimicrob Agents Chemother 2011;55:1485-93.

29. Kim SY, Park YJ, Yu JK, Kim YS. Aminoglycoside susceptibility profiles of Enterobacter cloacae isolates harboring the aac (6)-Ib gene. Korean J Lab Med 2011;31:279-81.

30. Leclercq R, Cantón R, Brown DF, Giske CG, Heisig P, MacGowan AP, et al. EUCAST expert rules in antimicrobial susceptibility testing. Clin Microbiol Infect 2013;19:141-60.

31. Almaghrabi R, Clancy CJ, Doi Y, Hao B, Chen L, Shields RK, et al. Carbapenem-resistant Klebsiella pneumoniae strains exhibit diversity in aminoglycoside-modifying enzymes, which exert differing effects on plazomicin and other agents. Antimicrob Agents Chemother 2014;58:444351.

32. Jakobsen L, Sandvang D, Jensen VF, Seyfarth AM, Frimodt-Møller N, Hammerum AM. Gentamicin susceptibility in Escherichia coli related to the genetic background: problems with breakpoints. Clin Microbiol Infect 2007;13:830-2. 
Lee $\mathbf{M}$, et al.

K. pneumoniae ST307 promiscuous plasmids

Supplemental Data Table S1. Primer for PCR identification of resistance determinants.

\begin{tabular}{|c|c|c|c|}
\hline Gene & Primer & Sequence & PCR size (bp) \\
\hline \multirow[t]{14}{*}{$\beta$-lactamase } & VIM_F & ATTGGTCTATTTGACCGCGTC & 780 \\
\hline & VIM_R & TGCTACTCAACGACTGAGCG & \\
\hline & IMP_F & CATGGTTGGTGGTTCTTGT & 488 \\
\hline & IMP_R & ATAATTGGCGGACTTTGGC & \\
\hline & NDM_F & TGGAATTGCCСАATATTATGC & 813 \\
\hline & NDM_R & TCAGCGCAGCTTGTCGGCCATGC & \\
\hline & OXA-48_F & TTGGTGGCATCGATTATCGG & 743 \\
\hline & OXA-48_R & GAGCACTTCIITGTGATGGC & \\
\hline & GES_F & ATGCGCTTCATTCACGCAC & 863 \\
\hline & GES_R & CTATTTGTCCGTGCTCAGGA & \\
\hline & KPC-2_F & ATGTCACTGTATCGCCGTCT & 893 \\
\hline & KPC-2_R & ПTTCAGAGCCTTACTGCCC & \\
\hline & Kpc-_F & CTGTCTTGTCTCTCATGGCC & 795 \\
\hline & Kpc_R & CCTCGCTGTGCTTGTCATC & \\
\hline \multirow[t]{8}{*}{ ESBLS } & TEM_F & ATGAGTATTCAACATTCCGT & 861 \\
\hline & TEM_R & TАССAATGCTTAATCAGTGA & \\
\hline & SHV_F & CCGGGTTATСТTATTGGTCGCT & 831 \\
\hline & SHV_R & TAGCGTGCCAGTGCTCG & \\
\hline & CTX-M-1_F & ACCGTCACGCTGTTGTTAGG & 819 \\
\hline & CTX-M-1_R & CAAGGTGACGATTTTAGCCG & \\
\hline & CTX-M-9_F & GTGCAACGGATGATGTTCG & 844 \\
\hline & CTX-M-9_R & АTGATTCTCGCCGCTGAAG & \\
\hline \multirow[t]{8}{*}{ AmpC } & ACT_F & TCGGTAAAGCCGATGTTGCGG & 302 \\
\hline & ACT_R & сTССACTGCGGCTGCCAGT & \\
\hline & ACC_F & AACAGCCTCAGCAGCCGGTTA & 346 \\
\hline & ACC_R & TTCGCCGCAATCATCCCTAGC & \\
\hline & CMY_F & TGGCCAGAACTGACAGGCAAA & 462 \\
\hline & CMY_R & ПтТTCCTGAACGTGGCTGGC & \\
\hline & DHA_F & AACTTCACAGGTGTGCTGGGT & 405 \\
\hline & DHA_R & CCGTACGCATACTGGCTITGC & \\
\hline \multirow[t]{8}{*}{ Aminoglycoside } & rmtA_F & CCCCATCGTGTGTTGGATAT & 635 \\
\hline & rmtA_R & GCACGCCCATACAGATGTTA & \\
\hline & rmtB_F & CGCATCCTGACTGAGG & 584 \\
\hline & rmtB_R & GGTGCGTGCAGCCTCT & \\
\hline & rmtD_F & CACAGCAAATCGCCCA & 532 \\
\hline & rmtD_R & CGGTCAGTGCGTAAAC & \\
\hline & armA_F & TGAAAGAGTCGCAACATTAAATGA & 590 \\
\hline & armA_R & CCATTGGTATAAAGCTAATGGATTG & \\
\hline \multirow[t]{8}{*}{ Fluoroquinolone } & qnrA_F & AGAGGATTTCTCACGCCAGG & 580 \\
\hline & qnrA_R & TGCCAGGCACAGATCTTGAC & \\
\hline & qnrB_F & GGAATTGAAATTCGCCACTG & 264 \\
\hline & qnrB_R & TITGCCGCCCGCCAGTCGAA & \\
\hline & qnrS_F & GCAAGTTCATTGAACAGGGT & 428 \\
\hline & qnrS_R & TCTAAACCGTCGAGTTCGGCG & \\
\hline & qepA_F & CCGACAGGCCCACGACGAGGATGC & 549 \\
\hline & qepA_F & TCGGCGGCGTGTGCTGGAGTTCT & \\
\hline \multirow[t]{4}{*}{ Outer membrane protein } & ompK35_F & AACTTATTGACGGCAGTGGC & 1,054 \\
\hline & ompK35_R & TTGGTAAACGATACCCACGG & \\
\hline & ompK36_F & GCAGTGGCATAATAAAAGGCA & 1,080 \\
\hline & ompK36_R & ACTGGTAAACCAGGCCCAG & \\
\hline
\end{tabular}

